\title{
Chemopreventive Agent
}

National Cancer Institute

\section{Source}

National Cancer Institute. Chemopreventive Agent. NCI Thesaurus. Code C1892.

A natural or synthetic substance, such as a drug, vitamin, hormone, plant product or food supplement, that may reduce the risk of developing or recurrence of tumor formation. Chemopreventive agent may work at different stages and may inhibit the initiation, promotion, transformation, and/or progression of the malignant process. 